\title{
DRAGON ON TERRORISM: ASSESSING CHINA'S TACTICAL GAINS AND STRATEGIC LOSSES POST-SEPTEMBER 11
}

Mohan Malik

"

October 2002 